\title{
Wearable Biosignal Monitoring System for Newborns
}

\author{
Paolo Perego, Giuseppe Andreoni, \\ Design Dept. Politecnico di Milano, \\ Milan, Italy \\ \{giuseppe.andreoni, paolo.perego\}@polimi.it
}

\author{
Rinaldo Zanini, Roberto Bellù \\ Az.Osp. Provincia di Lecco "A. Manzoni” \\ Lecco, Italy \\ \{r.zanini, r.bellu\}@ospedale.lecco.it
}

\begin{abstract}
In the last decade, the trends in the development and management of national healthcare service are focused telemedicine with the aim of cost reduction. Telemedicine can be useful to reduce cost thanks to early de-hospitalization and diagnosis. These development drivers can be achieved only through the use of new technology oriented to the production of low cost product, which can be integrated with existing system in order to improve monitoring quality and possibility. The application of these technologies on sensible aspects, as newborn monitoring, has to face the main issue of user acceptability. This paper shows the application of a clinical protocol to a wearable system for monitoring the first two hours of life. The results underline the capability of textile electrodes for wearable monitoring and show a great acceptability by both parents and nursing staff.
\end{abstract}

Keywords-component; wearable monitoring; newborns, acceptability test, textile electrodes, smart garment

\section{INTRODUCTION}

Despite the term "Wearable Computer" was coined in 1960s, and the first smart textile in 1996 [1], only in the last three years, with the development of new micro and low energy electronics components, as well as in information processing and wireless communication, smart wearable devices have made their appearance on the market. In the last three years, wearable devices became one of the most growing market and many of the major technological company invest in developing wearable technology, especially activity tracker and heart-rate monitor. But only few attempts have been done using wearable devices for medical purposes [2]. However, thanks to their features of wearability and non-invasiveness, these kinds of devices, and particularly the smart garments, can be useful for non-invasive diagnosis and treatment of chronic disease.

On of the most promising field of application of smart garment is baby monitoring [3]. Smart garments allow for monitoring without modifying the state of the baby and the acquired signals themselves. One of the most sensible periods of time in the first day of life of a newborn is the first 2-4 hours of life. When the baby is born, for the first 2-4 hours there is no monitoring active; the baby, after a rapid cleaning procedure and first checkup, is given naked to the mother who put $\mathrm{him} / \mathrm{her}$ on her breast. The skin-to-skin contact facilitate the maternal bonding [4] which consists in different aspect: thermoregulation of the baby, the kangaroo care [5], cognitive bond, etc. In this period of time, normally there is no ongoing monitoring in order to favor the bonding between mother and child; a discontinuous monitoring is usually implemented through a common visual recognition by the nursery staff. In this time, when the baby is in a transient period from pre- to post-natal, a continuous monitoring of heart rate is clinically recommendable to prevent critical situations [6] but up to now no systems are available for this purpose in an unobtrusive way. The proposed monitoring systems aims at solving this clinical need and to impact on the organization of the work of nursery staff, and increase the quality of the health service delivered through the use of a continuous quantitative monitoring, rather then a qualitative observation repeated over time in a discontinuous manner. This kind of monitoring system requires being the least invasive as possible under different aspects:

- $\quad$ for the medical procedure;

- for the baby;

- for the mother.

The use of smart garments with textile comfortable electrodes [7] is advisable for these applications [3]. Despite this, the introduction of a new monitoring procedure in a sensitive environment like the delivery room can be very stressful. For this reason, an acceptability study is mandatory. This work presents the study of the compliance, the acceptability and the reliability of a new monitoring system applied in the first 2-4 hours of life of a newborn during the bonding procedure.

\section{WeARABLE Monitroing SyStem}

The wearable monitoring system used for acceptability test is composed by:

- a wearable system;

- a Bluetooth to Ethernet Access Point;

- a control station.

The wearable system consists in two parts (figure 1):

- the smart garment;

- the monitoring unit, i.e. a Bluetooth wearable device;

The smart garment is the sensible part; it consists in a small shawl (produced in two sizes in order to fit all the babies), which includes on the sleeves two textiles electrodes. These electrodes allow for recording the ECG I-lead. The garment is hypoallergenic, soft and comfortable; its shape allows for a non-invasive measurement, and at the same time, the skin-toskin contact with the mother, without limiting the activities of caregivers. The ECG signal acquired by the textile electrodes is bring on the back of the baby through textiles wires. On the back there are two automatic snaps to connect the device for signal monitoring. The device is placed on the back to avoid comfort problem and accidental disconnection due to the baby's position remains hugged to the mother during the $2 / 4$ hours bonding. This device allows for acquiring continuously for 17 hours one ECG lead and tri-axis accelerations at sample frequency of $128 \mathrm{~Hz}$. All the acquired data are de-noised by the electronics. Since the device must be used on newborns, it is 
advisable to use medical certified and low power wireless transmission systems. Therefore our device uses Bluetooth 2.1+EDR which allows for transmitting signals to the access point through a SPP profile connection, which allow a relative high data rate. The access point consists of a commercialized device (Bluegiga Long Range Bluetooth Access Point 3241), which allow for get data from a Bluetooth SPP connection and re-sent them packet on a specific TCP-IP port. The TCP-IP connection, for convenience reason, has been structured by means of router on a $\mathrm{Wi}-\mathrm{Fi}$ connection.

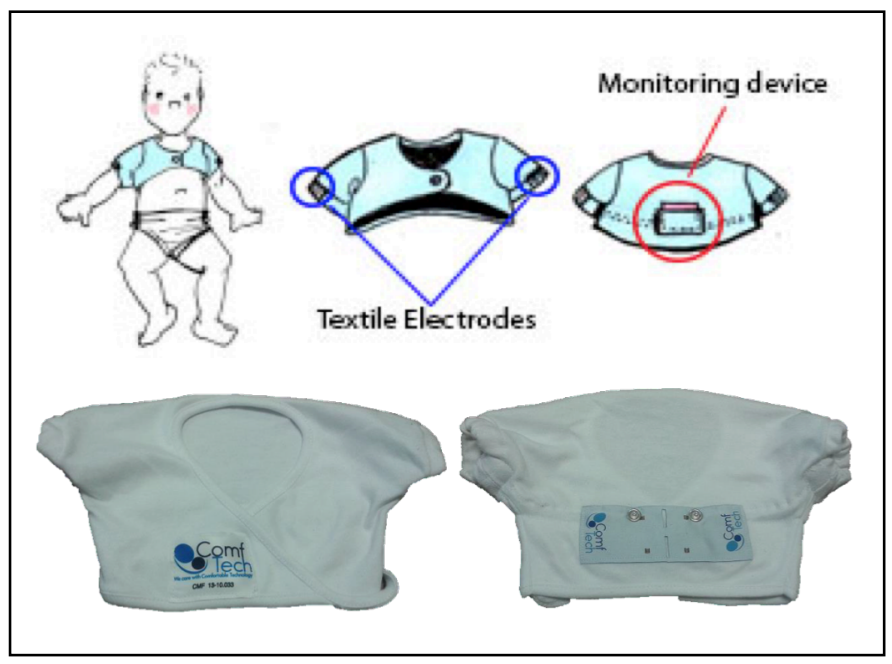

Figure 1. The wearable smart garment and the back position of the monitoring unit.

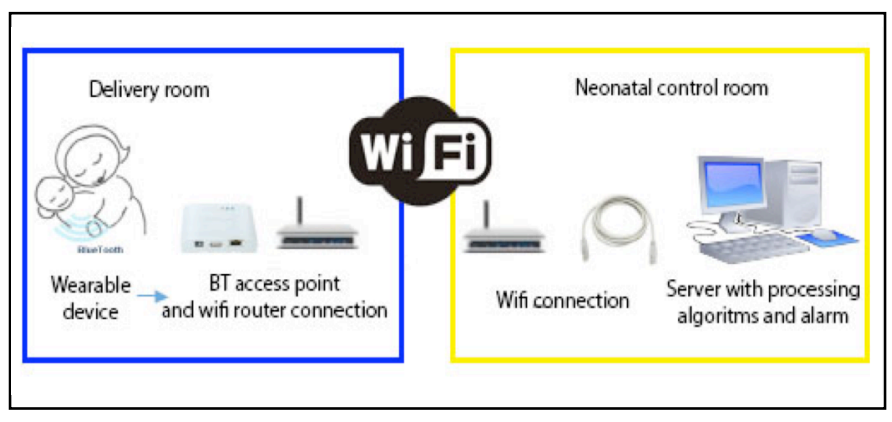

Figure 2. The complete scheme of the wearable monitoring system.

The server receives the packets in the same format sent from the Bluetooth device; this allows using either a computer or a mobile device to receive the data. All the data are digitally filtered to remove $50 \mathrm{~Hz}$ noise and then processed in order to extract hearth-rate (HR) and an estimation of the breath-rate (BR) through EDR algorithm [8] which are standard indexes of health status.

Data are stored locally and can be processed in real time to generate an alarm in case of:

- HR and BR out of the normal range of values;

- absence of ECG signal;

- Bluetooth or Ethernet connection problems.

The nurse can visualize the alarm on the server and intervene in case of need.

\section{THE ClinicAl TRIAL}

This work aims to study the reliability and the acceptability of a wearable system for monitoring the first $2 / 4$ hours of life. The purposes of the study can be summarized in:

- Verify the compliance of the medical staff;

- Verify the compliance of mother and relatives;

- Verify the quality of acquired signals.

The study of reliability and acceptability has been structured as follows:

1. The mother and the partner sign the written informed consent at the time of acceptance into the delivery room.

2. After the birth, the baby is rapidly cleaned and checked with standard neonatal control (Apgar score at 1 and 10 minutes).

3. The nurse dresses the baby with the sensorized shawl.

4. The nurse switches on and connect the monitoring device to the shawl on the back of the baby.

5. The baby is given to the mother for the bonding time (usually 2 hours).

6. The nurse, in the neonatal control room, remotely activates the acquisition.

7. The Central Monitoring Station (server) in the neonatal control room records and store ECG and motion data.

The Central Monitoring Station can be connected up to three different monitoring devices in order to be able to monitor all the delivery rooms at the "A. Manzoni" Hospital of Lecco, where the clinical study where carried out. The alarm function was not enabled for this first clinical trial: alarm/warning conditions were computed through an offline processing to verify data quality and possible criticalities.

For hygienic reasons, the sensorized shawl was created as single-use disposable garment. The electronics device case instead is made by soft material in order to be comfortable, easily disinfected and reusable.

The study has been divided into two parts.

The first part regards the reliability of the system, and in particular the signal quality study. The recorded and locally stored data allow a statistics on the signals to verify the quality of the acquired data. The signal quality regards only the ECG signal. ECG signal has been divided in good signal and nogood signal to check the percentage of useful data and emphasize the presence of artifacts and noise.

The second part consists in three questionnaires to collect data on the system usage. This allows for verifying the compliance, usability and acceptability. The questionnaires are applied to all the actors: mothers, partners and nursery staff; the first two are considered indirect users because they use the system but they don't interact actively with it; the last one is the direct user because is the nurse or the midwife who set and applies the system. The questionnaires included four different aspects of acceptability: ergonomics and general usability; general reliability; perceived safety; overall satisfaction. All these aspects were investigated with two to five questions with a subjective evaluation organized in a rank scale ranging from 
1 (absolutely negative) to 10 (excellent) with a neutral assessment set at 5 .

\section{RESUltS AND Discussion}

30 newborns with gestational age between 38 and 41 weeks (mean $=39.9 \mathrm{w}, \mathrm{SD}=1.0)$ participated to the study. 28 out of 30 were vaginal deliveries, and the other 2 ones were caesarean sections. Written informed consent was obtained from subjects and families according to the Declaration of Helsinki. The Ethical Committee of the "A. Manzoni" Hospital in Lecco approved the research.

Figure 3 reports the results of the overall system. Two tests were interrupted for a sudden clinical problem of the mothers (who needed clinical assistance thus breaking the bonding procedure) and one was interrupted due to complication of the newborn. One trial was withdrawn for a sudden renounce of the mother when the newborn was to be prepared for the monitoring even if she had already signed the informed consent. However we included these tests in the statistical analysis (figure 3), that is divided into two sections: technical reliability analysis and ergonomic analysis.

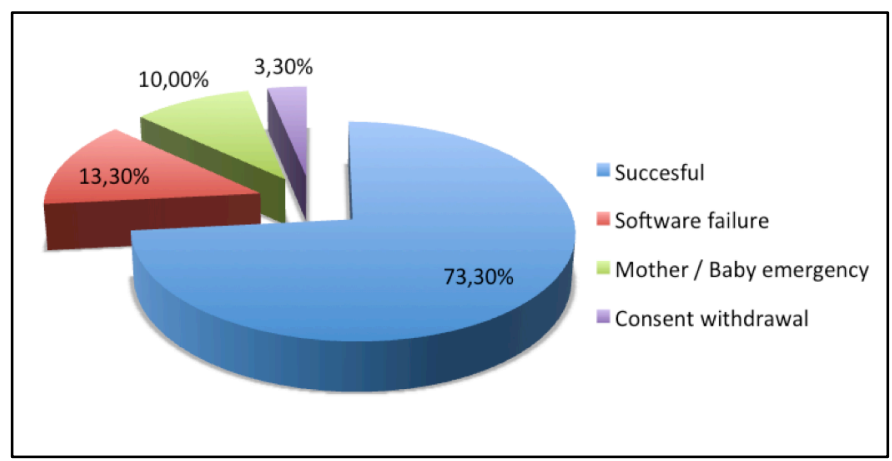

Figure 3. Summary of the validation test results.

Technical reliability analysis

The system and the related analysis is divided according to the three components:

1. The wearable biomedical sensor composed by the sensorized garment and the unit for ECG and motion measurement and transmission;

2. The wireless gateway and the related software;

3. The control station and the related software.

Starting from this last component, 4 trials out of 30 failed for a software problem in the Control Room station. The failure in the initialization of the acquisition was due to a library element of the new Windows OS; the issue has been identified and corrected for future developments and experiments.

In relation to the gateway component no problems were faced and no trials failed for connection problems.

Regarding the wearable biomedical sensor the clinical trial evidenced the following results:

- 21 successful recordings;

- 1 trial failed for low battery of the monitoring device;

In the only case of failure due to exhaust monitoring device (low battery), the problem is not amenable to the system itself, but to the incorrect deployment of charging of the device.
In a preliminary analysis of the ECG signals we noted that in two cases a long absence of ECG signal occurred. From the notes in the corresponding questionnaires and from data observation, we found a detachment of the snaps that connect the device to the sensorized shawl. This problem was caused by the mother who cradling the newborn. This situation is not critical because it would have been easily detected by the alarm to the nurse if it had activated. In this case a prompt intervention of the nurse would detect and solve the detachment of the monitoring unit, so resolving the problem. For this reason we excluded these recordings from the following analysis.

The main and crucial point is the quality of the recorded ECG signal (an example in figure 5) to compute HR correctly. Table 1 shows the results of the quantitative analysis for signal quality. The ECG signal was divided into high-quality and lowquality signal, i.e. portion of signal were the HR computation is impossible or requires fine processing because of:

- $\quad$ signal absence;

- $\quad$ noise and artifacts.

A.

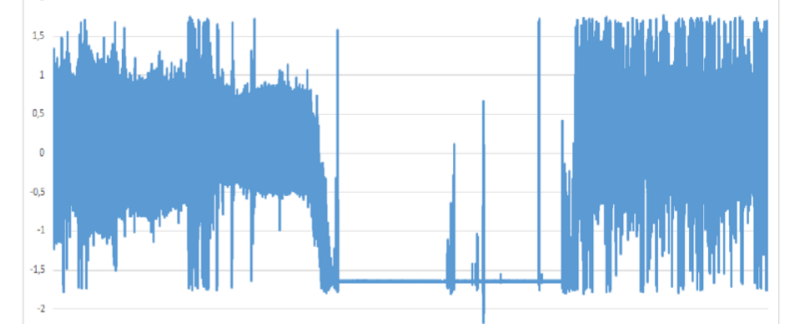

B.

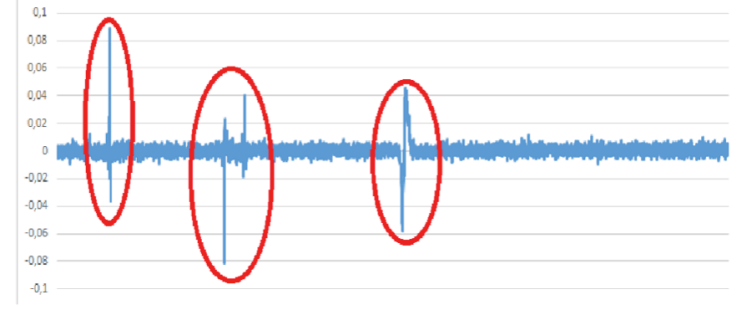

Figure 4. A. Signal interruption caused by the posting of snap buttons; B. Signal artifact caused by the movement of the electrodes to the skin.

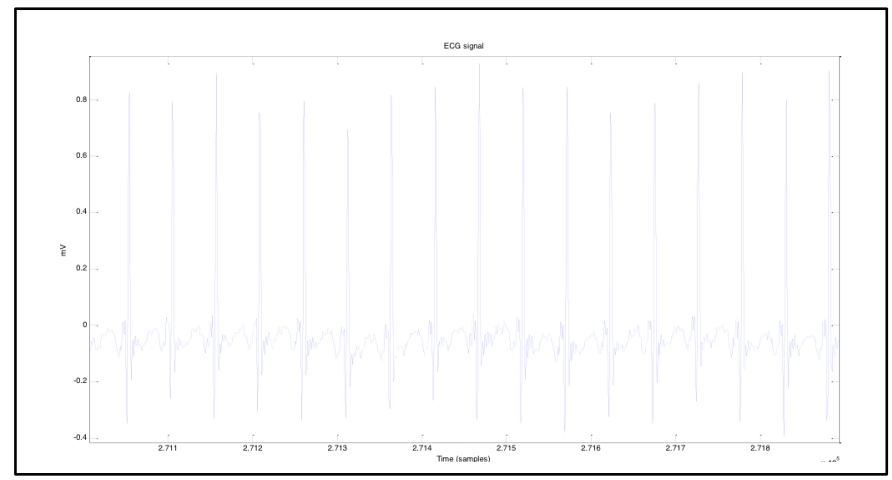

Figure 5. Example of ECG signal quality from the wearable sensors in newborn 10204 
Data in table 1 show the results from 19 considered trials for the analysis of the performance of the wearable biomedical sensor: the average percentage of high-quality signal is $75.8 \%$ $\pm 18.9 \mathrm{SD}$ (median 85.6\%). However, in this analysis it should be considered that part of the low-quality signal is due to the absence of signal (as shown in figure 4A) again for a partial (in time and recovered during the acquisition by the direct intervention of the mother) detachment of the snaps connecting the garment and the monitoring unit.

TABLE I. RESULTS OF SIGNAL QUALITY TESTS

\begin{tabular}{|c|c|c|c|}
\hline \multirow[b]{2}{*}{ ID } & \multicolumn{3}{|c|}{ Signal Quality test } \\
\hline & $\begin{array}{c}\text { Acquisition time } \\
\text { (sec) }\end{array}$ & $\begin{array}{c}\text { High-Quality } \\
\text { signal (sec) }\end{array}$ & $\%$ \\
\hline 1 & 5475 & 3685 & $67 \%$ \\
\hline 2 & 2888 & 2703 & $94 \%$ \\
\hline 3 & 8364 & 5340 & $64 \%$ \\
\hline 4 & 3699 & 1873 & $51 \%$ \\
\hline 5 & 7382 & 6654 & $90 \%$ \\
\hline 6 & 6793 & 5979 & $88 \%$ \\
\hline 7 & 892 & 818 & $92 \%$ \\
\hline 8 & 6533 & 3673 & $56 \%$ \\
\hline 9 & 920 & 555 & $60 \%$ \\
\hline 10 & 5867 & 1766 & $30 \%$ \\
\hline 11 & 6730 & 5964 & $89 \%$ \\
\hline 12 & 4380 & 3287 & $75 \%$ \\
\hline 13 & 6170 & 5263 & $85 \%$ \\
\hline 14 & 3808 & 3654 & $96 \%$ \\
\hline 15 & 6085 & 5619 & $92 \%$ \\
\hline 16 & 8795 & 7555 & $86 \%$ \\
\hline 17 & 1026 & 548 & $53 \%$ \\
\hline 18 & 4182 & 3214 & $77 \%$ \\
\hline 19 & 5264 & 4535 & $86 \%$ \\
\hline
\end{tabular}

Finally, in most acquisitions, there are some artifacts generally due to a short-time bad connection of the electrodes to the skin of the baby or by sudden movements. These events typically have a limited duration ( $<10$ seconds): this will not generate an alarm and therefore clinicians don't consider them. Thus by including also this portions of recordings into the high-quality signal the final reliability of the ECG signal raises up to $89.8 \% \pm 10.1 \mathrm{SD}$ (median $=94.8 \%$ ).

\section{Ergonomic Analysis}

The questionnaires allow for qualitative evaluating the aspects of ergonomic, and separately the perceived quality of the software. The results show that wearability of the sensor and usability of the software are largely positive (mean $=7.45$ $\pm 1.94 \mathrm{SD}$ ). Mothers and partners show a more positive acceptance of the wearable monitoring system (mean $=8.7, \pm$ 1.56 SD). Instead at the beginning of the clinical trial, the department staff has expressed some reservations on the adoption of this system in the current hospital process. A greater confidence was given to in the traditional monitoring respect to the new wearable system. This could be due to the strong innovation factor bringing by this system itself, and to the software problems faced in some trials. After this period a significant improvement of the scores is shown.

The safety perception of the mothers and partners are very positive, this shows that this kind of products could have a great impact in the hospital procedure in terms of perceived safety and quality (mean $=8.58 \pm 1.79 \mathrm{SD})$.

\section{CONCLUSION}

From the reliability point of view the wearable biomedical sensors provide excellent results while the wireless communication and control room software have shown bugs now identified and solved. From the point of view of user compliance and acceptance, the clinical trial shows very positive scores in particular by mothers and relatives this indicating the high usability and high expectation from the introduction of such a system in hospital practice. Nurses and midwives started with a certain degree of skepticism but turned into good acceptance and better scores than the standard procedures after a short training period.

\section{ACKNOWLEDGMENTS}

This work was partially supported by grant from Regione Lombardia (Programma Ergon) to the companies that provided the systems: ComfTech s.r.l for the wearable biomedical systems, Beta80 S.p.A. for the control room station, and Ab.Acus s.r.l. for questionnaire reporting. The authors want to thank the "A. Manzoni" Hospital of Lecco clinical staff of the Dept. and particularly all the nurses and midwives for the precious help during the clinical test. Thanks also to Eng. Maribel Eriksson for her help in data processing.

\section{REFERENCES}

[1] Georgia Tech Wearable Motherboard ${ }^{\mathrm{TM}}$ : The Intelligent Garment for the 21st Century, 2002, http://www.gtwm.gatech.edu/

[2] C. Bellos, A. Papadopoulos, R. Rosso, and D.I. Fotiadis, Clinical validation of the CHRONIOUS wearable system in patients with chronic disease, Proceeding at 35th Annual International Conference of the IEEE EMBS, Osaka, Japan, 3 - 7 July, 2013.

[3] P. Perego, A. Moltani, M. Fusca and G. Andreoni, Multi-parametric wearable systems for sports and homecare, Proceeding at pHealth, Porto, Portugal, 26-29 June, 2012.

[4] D.E. Eyer, Mother-infant bonding. Human Nature, 5(1),(2004): 69-94.

[5] V.L. Dodd, Implications of kangaroo care for growth and development in preterm infants $\}$, Journal of Obstetric, Gynecologic, and Neonatal Nursing 34.2 (2005): 218-232.

[6] J.C. Becher, S.B. Shetty. and J.L. Andrew. Unexpected collapse in apparently healthy newborns-a prospective national study of a missing cohort of neonatal deaths and near-death events, Archives of Disease in Childhood-Fetal and Neonatal Edition 97.1 (2012): F30-F34.

[7] G. Andreoni, A. Fanelli, I. Witkowska, P. Perego, M. Fusca, M. Mazzola and M. G. Signorini, A validation protocol for wearable sensors: an application roadmap for textile electrodes, IEEE Transactions on biomedical engineering, 1(2012) 1-7.

[8] Moody, G. B., Mark, R. G., Bump, M. A., Weinstein, J. S., Berman, A. D., Mietus, J. E., Goldberger, A. L., Clinical validation of the ECGderived respiration (EDR) technique. (1986). Group, 1(3). 